\title{
A critical appraisal on wavelet based features from brain MR images for efficient characterization of ischemic stroke injuries
}

\author{
Karthik. $\mathrm{R}^{*}$ and Menaka. $\mathrm{R}^{+}$ \\ * Assistant Professor, School of Electronics Engineering, VIT University, Chennai, India. \\ Email: karthikramamurthy@ymail.com; r.karthik@vit.ac.in \\ + Associate Professor, School of Electronics Engineering, VIT University, Chennai, India. \\ Email:menaka.r@vit.ac.in
}

Received 27th Aug 2015; accepted 24th Sep 2016

\begin{abstract}
Ischemic stroke is a severe neuro disorder typically characterized by a block inside a blood vessel supplying blood to the brain. It remains the third leading cause of death, after a heart attack and cancer. Computed Tomography (CT) and Magnetic Resonance Imaging (MRI) were the major imaging modalities used in diagnosing this disorder. While the CT imaging can be used at the primary stage, MRI proves to be a necessary aid for progressive diagnostic planning in the treatment of stroke injuries. Developing a fully automatic approach for lesion segmentation is a challenging issue due to the complex nature of the lesion structures. This research aims at examining the properties of such complex structures. It analyses the characteristics of the normal brain tissues and abnormal lesion structures using a three-level wavelet decomposition procedure. Four different wavelet functions namely Daubechies, Symlet, Coiflet and De-Meyer were applied to the different datasets and the resulting observations were examined based on their feature statistics. Experiments indicate that the feature statistics obtained using the Daubechies and the De-Meyer wavelets were able to distinguish between normal brain tissues and abnormal lesion structures.

Key Words: Ischemic Stroke, Watershed Transformation, Discrete Wavelet, Feature Statistics, MRI, Segmentation, Feature Extraction, Automated Diagnosis
\end{abstract}

\section{Introduction}

A Computer-Aided Detection (CAD) scheme can act as a vital aid to recognize the abnormal variations that clinicians may overlook sometimes and thus it improves the accuracy of the disorder prediction. Incorporating mathematical concepts into CAD models has made certain progress in the field of radiological science [1-2]. Automatic computer aided diagnosis of various disorders has been progressing rapidly in the past two decades. Among the various disorders, stroke takes the third place after heart attack and cancer for being the reason for death [3]. Computed Tomography (CT) and Magnetic Resonance Imaging (MRI) were the two major imaging techniques used for diagnosing the stroke disorder. Due to the rapid advancement in the diagnostic imaging modalities, the identification of infarct lesion is turning out to be progressively more feasible. However, it is very complex due to the arbitrary size, shape and location of the lesion structures. Manual segmentation in combination with some statistical measures has been broadly utilized in the past few years to explore the diverse aspects of stroke injuries.

Correspondence to: karthikramamurthy@ymail.com

Recommended for acceptance by João Manuel Tavares

http://dx.doi.org/10.5565/rev/elcvia.804

ELCVIA ISSN: $1577-5097$

Published by Computer Vision Center / Universitat Autonoma de Barcelona, Barcelona, Spain 
Despite of the fact that a wide range of segmentation approaches were introduced namely thresholding [4-5], region-based approaches [6-7], and clustering [8-9], these methods were reported to be complex when adopted for detecting the lesion structures. This is due to the fact that lesions usually have quite similar intensity with respect to some normal tissues. For instance, in T1- weighted MRI, an infarct lesion was indicated with intensities equivalent to those of Gray Matter (GM) or Cerebrospinal Fluid (CSF). Hence, a standard segmentation method might relate them together instead of treating it separately.

Several approaches were presented in the recent years for detecting the ischemic lesions using semiautomated techniques. These approaches model the texture properties of the Region of Interest (ROI). The analysis of texture essentially describes a wide range of image analysis approaches which would characterize the variation in the surface patterns or intensities. This is highly suited for segmenting the lesion structures and characterizing it. In addition, it serves as a vital aid for longitudinal monitoring of disorders or recoveries [10].

Tan et al. developed a method for contrast enhancement of CT images in order to detect the lesion structures due to stroke [11]. Chawla et al. presented a two-level classification system using an automatic histogram to localize the ischemic lesions [12]. Bagher-Ebadian et al. introduced a classification system using adaptive (two-level) Gaussian mixture model and probabilistic neural network [13]. Dastidar et al. presented a semi-automated approach for segmenting the ischemic lesions using intensity histograms followed by region growing [14]. Nabizadeh et al. presented an approach for identifying the brain tumor regions using two different techniques namely Gabor wavelet features and statistical features [15]. Shuyu Yang et al. presented an approach for modeling of medical images using hybrid multi-level wavelet features [16].

Active contour models were also employed for segmentation of anatomical shapes from biomedical images [17]. Several variants of active contour based approaches were proposed in the last few years. A structural feature region-based active contour model focused on the level set method was presented for image segmentation by Qi Ge et al. [18]. A non-convex vector-valued model of Active Contour Without Edges (ACWE) was introduced for texture image segmentation [19]. Deformable models were also applied to MRI images to segment the tumor lesions [20].

Most of the above-discussed works were either manual or semi-automated approaches, leaving considerable overhead to the analyst or radiologist for fixing the parameters for analysis. Thus, this research aims to examine the properties of the lesion structures, which could be employed to address those issues. If the properties of the lesion structures were understood properly, then it could be further modeled as an automated system for segmenting the lesion structures.

The paper is structured as follows: Section 2 presents the complete details of the proposed approach for modeling the properties of the brain tissues. Section 3 presents the results obtained from the proposed method and the performance aspects of it. Section 4 presents the conclusion and possible future enhancements of the proposed work.

\section{Methodology}

The proposed approach employs skull stripping as the initial pre-processing step for eliminating the bony portions from the input MRI images. Later, a watershed-based segmentation procedure was applied to localize the lesion region [21]. From the extracted ROI, a three level wavelet decomposition has been carried out with different basis functions. Finally, statistical features were extracted to characterize the texture patterns of the normal and abnormal images. The overview of the proposed method appears in Figure 1. 


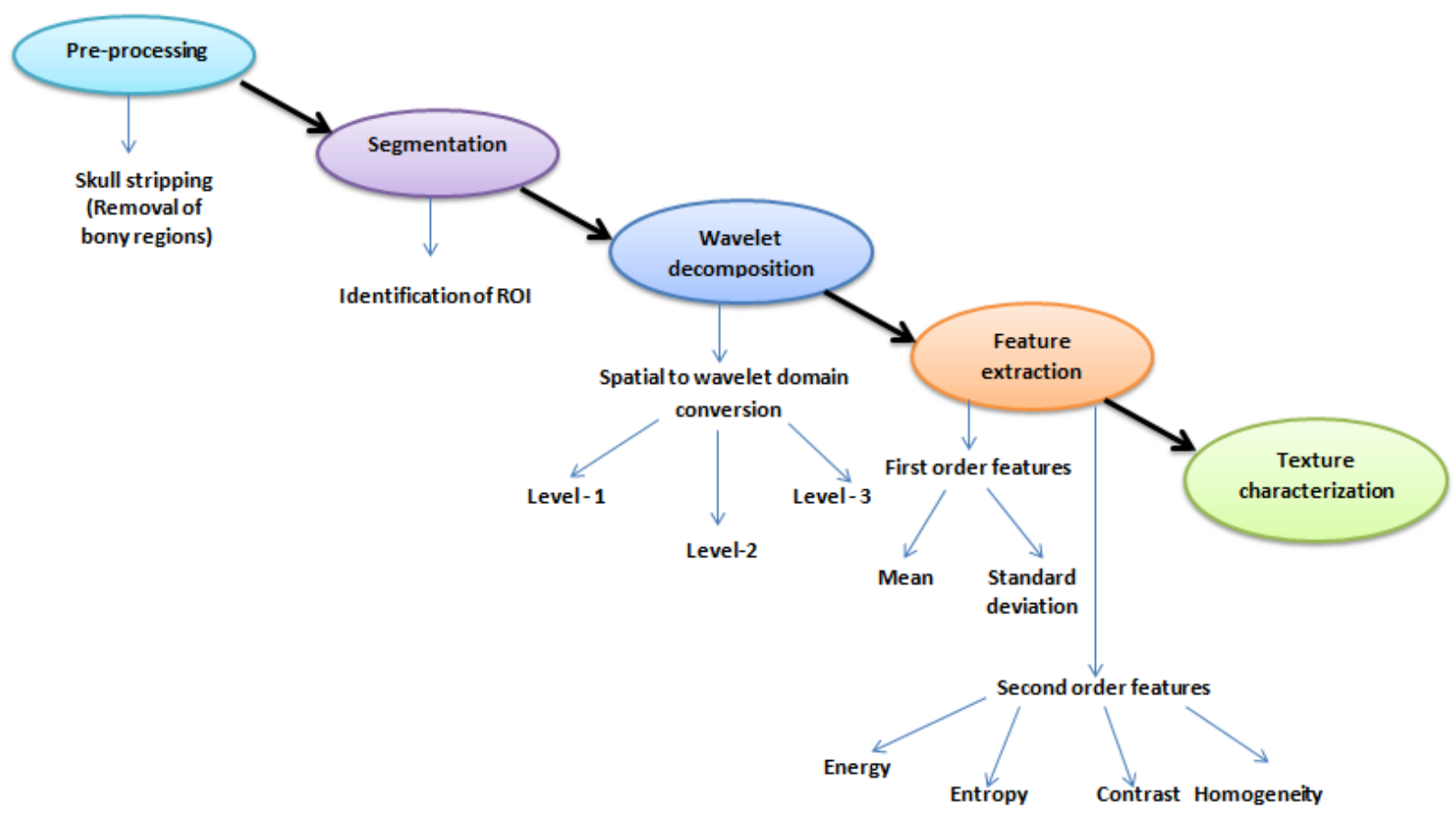

Figure 1: Overview of the proposed methodology.

\subsection{Data Acquisition}

The MRI datasets were collected from multiple online and offline sources. The online resources include the ISLES dataset provided as part of ISLES challenge held at Munich, Germany [22-23]. The other datasets were obtained from PBM Hospitals and Global Health City. A total of 45 datasets were used in this research, out of which, 25 had radiological evidence of ischemic stroke. The format of the input MRI Slices was in Neuroimaging Informatics Technology Initiative (NIfTI) and Digital Imaging and Communication in Medicine (DICOM).A Sample normal and abnormal MRI slice with ischemic and hemorrhagic stroke was presented in Figure 2.

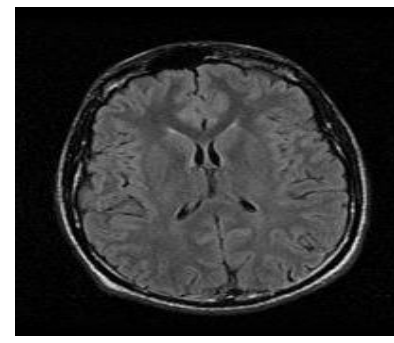

(a)

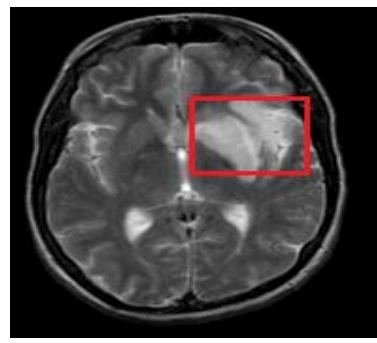

(b)

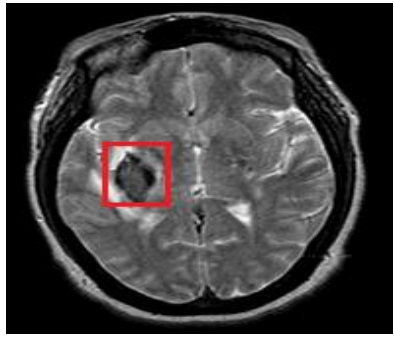

(c)

Figure 2: Normal MRI slice (b) MRI slice with ischemic stroke lesion (c) MRI Slice with hemorrhagic stroke lesion.

\subsection{Pre-processing}

The skull portion is the largest connected region in the brain image with higher bone density. This information could be utilized as an essential sign to remove the bony portions from the input image. Initially, the input image is converted into a binary image based on the threshold obtained for the skull region using global thresholding. i.e. a mask is formed by setting the pixels from the skull region to ' 0 ' 
and the remaining pixels as ' 1 '. This mask is convolved with the input image to remove the skull portion. The corresponding results are presented in Figure 3.

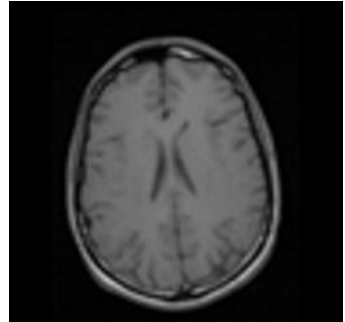

(a)

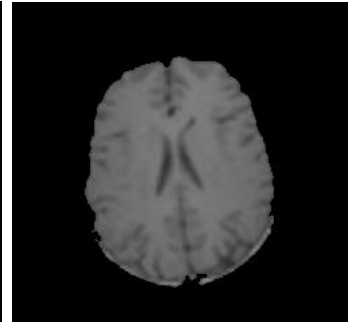

(b)

Figure 3: Pre-processing: a) Input Image and b) Skull stripped image.

\subsection{Segmentation}

The goal of the segmentation step is to identify and localize the ROI required for further processing. Watershed transformation has been widely applied to brain images in order to extract the abnormal regions from brain images [24]. This work involves watershed transform based segmentation procedure for identifying and localizing the lesion structures from the pre-processed images. This method considers the gray scale pixel intensities of the original image as the altitude of the water basin. The drops of water flow through the maxima of values and then reach the local minima. Thus, the watershed formed is equivalent to the boundary of the segmented regions. This segmentation procedure was applied to the input images and the resulting segmented regions were presented in Figure 4. It could be noted that, the lesion region were identified as local maxima for the abnormal slices and hence it was appropriately segmented. Similarly, for the normal images, the white matter content was predominately captured.

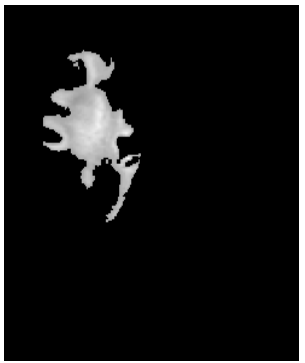

(a)

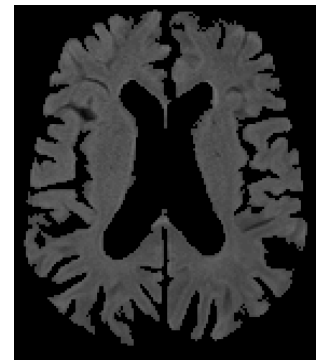

(b)

Figure 4: (a) Segmented lesion region from abnormal slice (b) Segmented region from normal slice.

\subsection{Wavelet decomposition}

The wavelet transform limits the energy of the signal in a combined space-scale domain. It decomposes an image into their elementary constituents across multiple scales [25]. A mother wavelet function is repeatedly dilated, shifted and convolved with the input data. Wavelet decompositions are extremely helpful for the characterization of point singularities and in the time-frequency analysis of nonstationary signals [26]. Wavelet analysis consists of decomposing an image into a hierarchical set of approximations and details. At each level, the signal is decomposed into low and high frequencies, and this decomposition halves the resolution since only half of the samples are retained to represent the complete signal. Two dimensional discrete wavelet transform leads to a decomposition of approximation coefficients at level ' $\mathrm{j}$ ' in four components. It is highly difficult to characterize the lesions which can be very small to be easily seen. In this research, lesions were characterized by the texture features computed from the Gray Level Co-occurrence Matrix (GLCM) in the wavelet domain.

In this work, four different wavelet functions were applied to the input MRI images for examining the properties of the lesion structures. The discrete wavelet transform of an image $f(x, y)$ of size $[M, N]$ is given in Equation 1 and 2. 


$$
\begin{aligned}
& W_{\varphi}\left(j_{0}, m, n\right)=\frac{1}{\sqrt{M N}} \sum_{x=0}^{M-1} \sum_{y=0}^{N-1} f(x, y) \varphi_{j_{0, m}, n}(x, y) \\
& W_{\gamma}^{i}(j, m, n)=\frac{1}{\sqrt{M N}} \sum_{x=0}^{M-1} \sum_{y=0}^{N-1} f(x, y) \gamma_{j . m, n}^{i}(x, y), i=\{H, V, D\}
\end{aligned}
$$

where ' $\mathrm{j}_{0}$ ' represents the starting scale, ' $\mathrm{W} \varphi\left(\mathrm{j}_{0}, \mathrm{~m}, \mathrm{n}\right)$ ' defines the approximation coefficients of $\mathrm{f}(\mathrm{x}, \mathrm{y})$ at scale ' $\mathrm{j}_{0}$ ', ' $W_{\gamma}^{i}(j, m, n)$ 'coefficients add horizontal ' $\mathrm{H}$ ', vertical ' $\mathrm{V}$ ' and diagonal ' $\mathrm{D}$ ' details for scales ' $\mathrm{j}$ $\geq \mathrm{j}_{0}$ '. The detail coefficients along the vertical, horizontal and diagonal directions capture the edge activity of the image and the approximation coefficients concentrate on the inner details of the image. Four different wavelet functions namely Daubechies, Symlet, De-Meyer, and Coiflet were applied to the input images. The scaling and wavelet functions applied with respect to each wavelet type are presented in Figure 5.In this research, the level of decomposition was varied from ' 1 ' to ' 5 ' and the results were analyzed. It was inferred that the results obtained after third level was not significant due to more deviation obtained between the original image and the approximated image at those levels. Hence the level of decomposition was maintained to be ' 3 '. The Daubechies wavelet decomposition for the segmented ROI was presented in Figure 6.
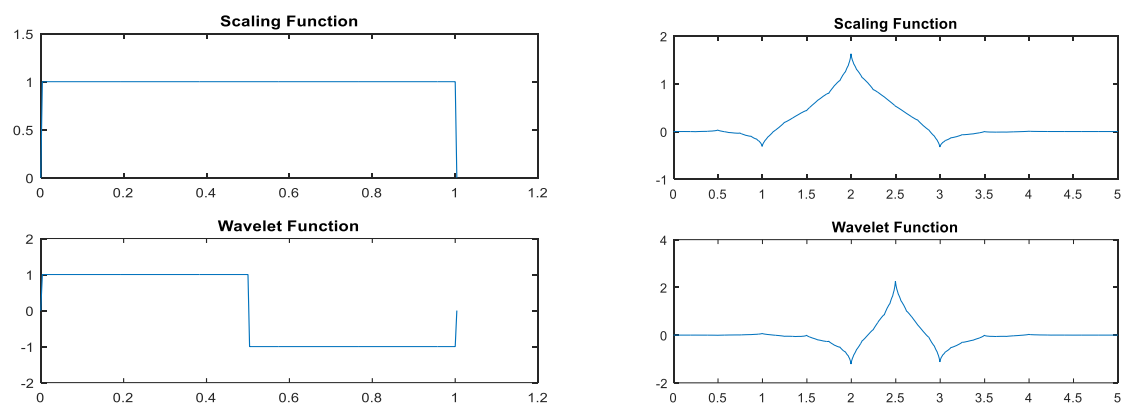

(a)
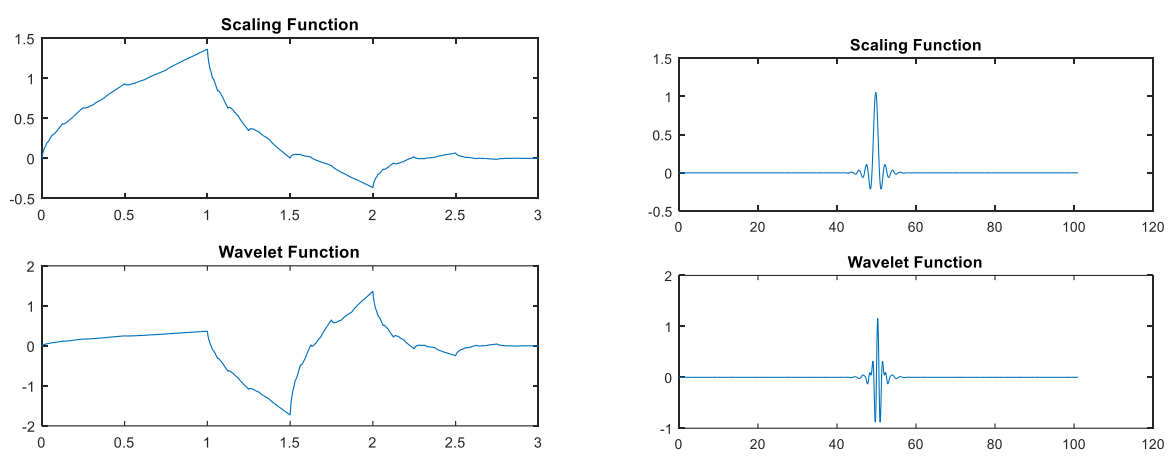

(c)

(d)

Figure 5: (a) Daubechies (b) Coiflet (c) Symlet (d) De-Meyer.

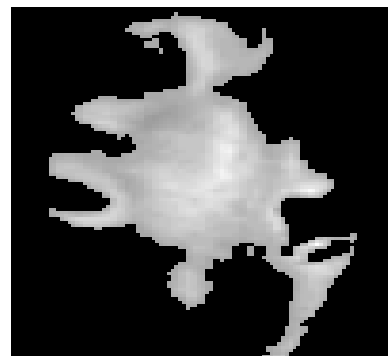

(a)

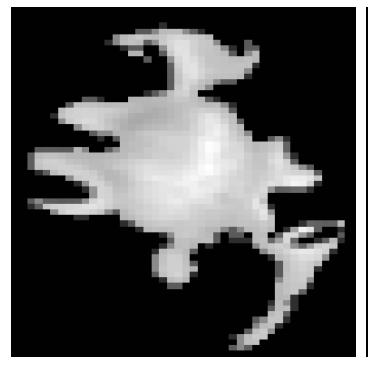

(b)

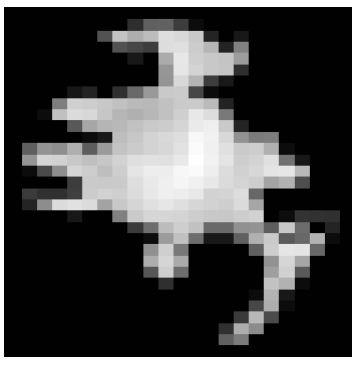

(c)

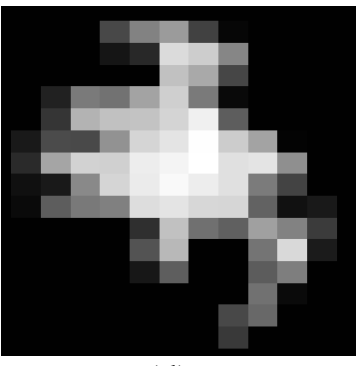

(d)

Figure 6: (a) Input image (b) Level-1 Approximation (c) Level-2 Approximation (d) Level-3 Approximation. 


\subsection{Feature extraction}

Once the wavelet decomposition has been completed, texture primitives should be extracted from it. It is characterized by the distribution of gray levels in a neighbourhood. To model the relationship between gray level values which add to the perception of texture, the Gray Level Co-occurrence Matrix (GLCM) is utilized in texture analysis. It is a 2D histogram which defines the occurrence of pixel pairs separated by a distance factor ' $\mathrm{d}$ '. The detailed description of this matrix was discussed in [15]. The same process is adopted in this work except that the input images were handled in wavelet domain instead of the spatial domain. The main objective of this research is to identify an optimal feature vector which could categorize the nature of the brain tissues. Hence, the prominent features which will reflect the textural relationships based on the neighbourhood pixels were alone considered for analysis. Four features namely energy, entropy, homogeneity and contrast parameters were extracted from the GLCM. These four features along with the mean and standard deviation have been taken into account for examining the properties of the brain tissues. The four features extracted from the co-occurrence matrix were illustrated in Equations 3 to 6. In these relations, $G$ is the maximum gray level of the image and $p(i, j)$ is the probability of the element located at the position $(i, j)$ in the wavelet domain.

\section{Energy}

It quantifies the uniformity of an image. For a uniform image with less variation in their details, the energy parameter will be equal to 1 .

$$
\text { Energy }=\sum_{\mathbf{i}=0}^{\mathrm{G}-1} \sum_{\mathbf{j}=0}^{\mathrm{G}-1}(\mathbf{p}(\mathbf{i}, \mathbf{j}))^{2}
$$

\section{Entropy}

This parameter presents the information content of an image.

$$
\text { Entropy }=\sum_{i=0}^{\mathrm{G}-1} \sum_{\mathbf{j}=0}^{\mathrm{G}-1} \mathbf{p}(\mathbf{i}, \mathbf{j}) . \log (\mathbf{p}(\mathbf{i}, \mathbf{j}))
$$

\section{Contrast}

The contrast parameter presents the amount of local differences in an image. It provides the level of variation in the contrast of the identified regions. If the variations are relatively higher in an image, then the contrast will consequently be high.

$$
\text { Contrast }=\sum_{\mathbf{n}=0}^{\mathrm{G}-1} \mathbf{n}^{2}\left(\sum_{\mathrm{i}=1}^{\mathrm{G}-1} \sum_{\mathbf{j}=1}^{\mathrm{G}-1} \mathbf{p}(\mathbf{i}, \mathbf{j})\right),|\mathbf{i}-\mathbf{j}|=\mathbf{n}
$$

\section{Homogeneity}

It provides the degree of nearness of the components in the GLCM with the diagonal of the same matrix. It indicates the uniformity of the contents present in the image.

$$
\text { Homogeneity }=\sum_{\mathrm{i}=0}^{\mathrm{G}-1} \sum_{\mathrm{j}=0}^{\mathrm{G}-1} \frac{\mathrm{p}(\mathrm{i}, \mathrm{j})}{1+|\mathrm{i}-\mathrm{j}|}
$$

These features were extracted for different datasets and the corresponding observations obtained were graphically presented in Figure 7 to 14. 

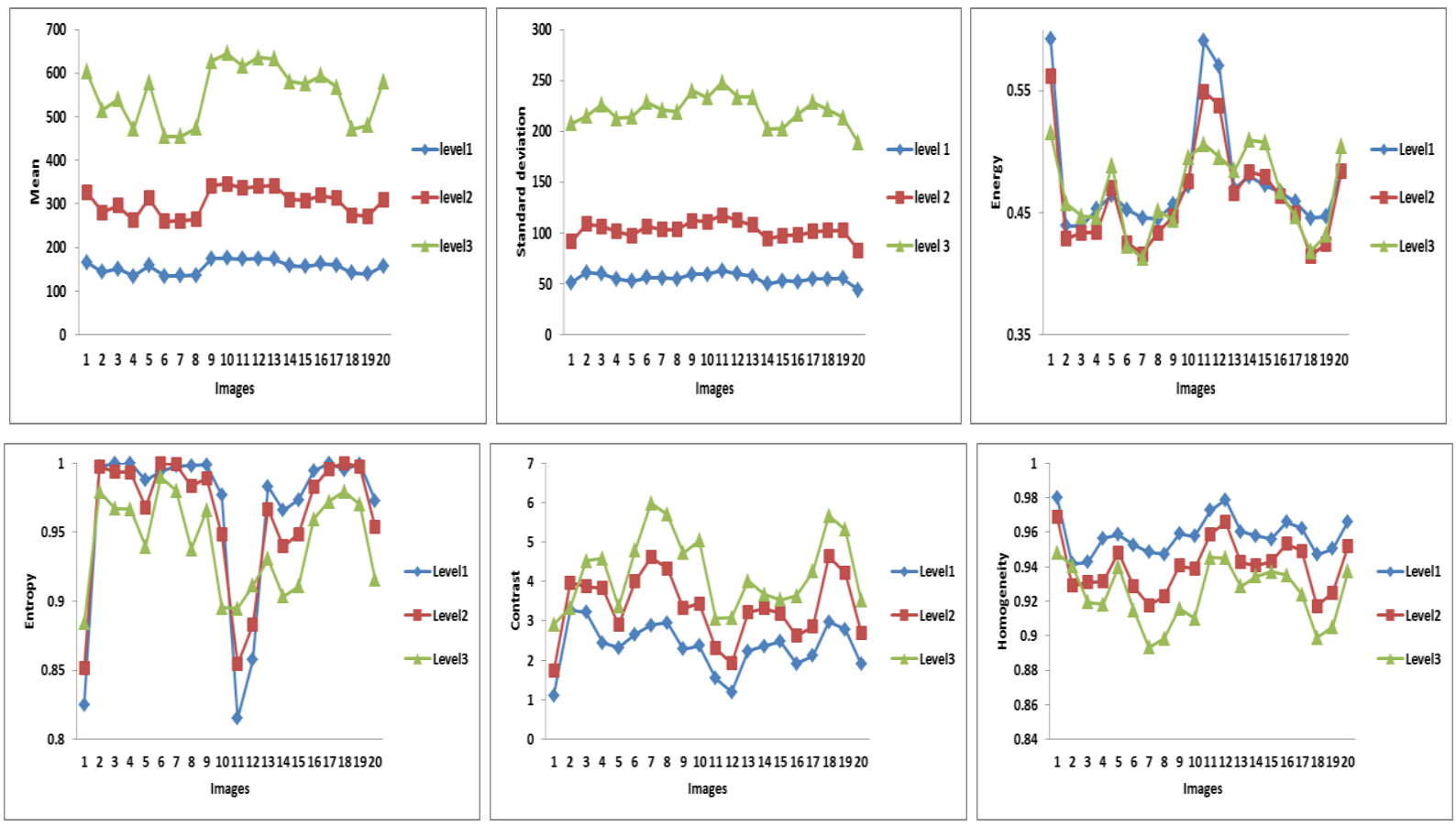

Figure 7: Daubechies wavelet based statistical features obtained for normal datasets
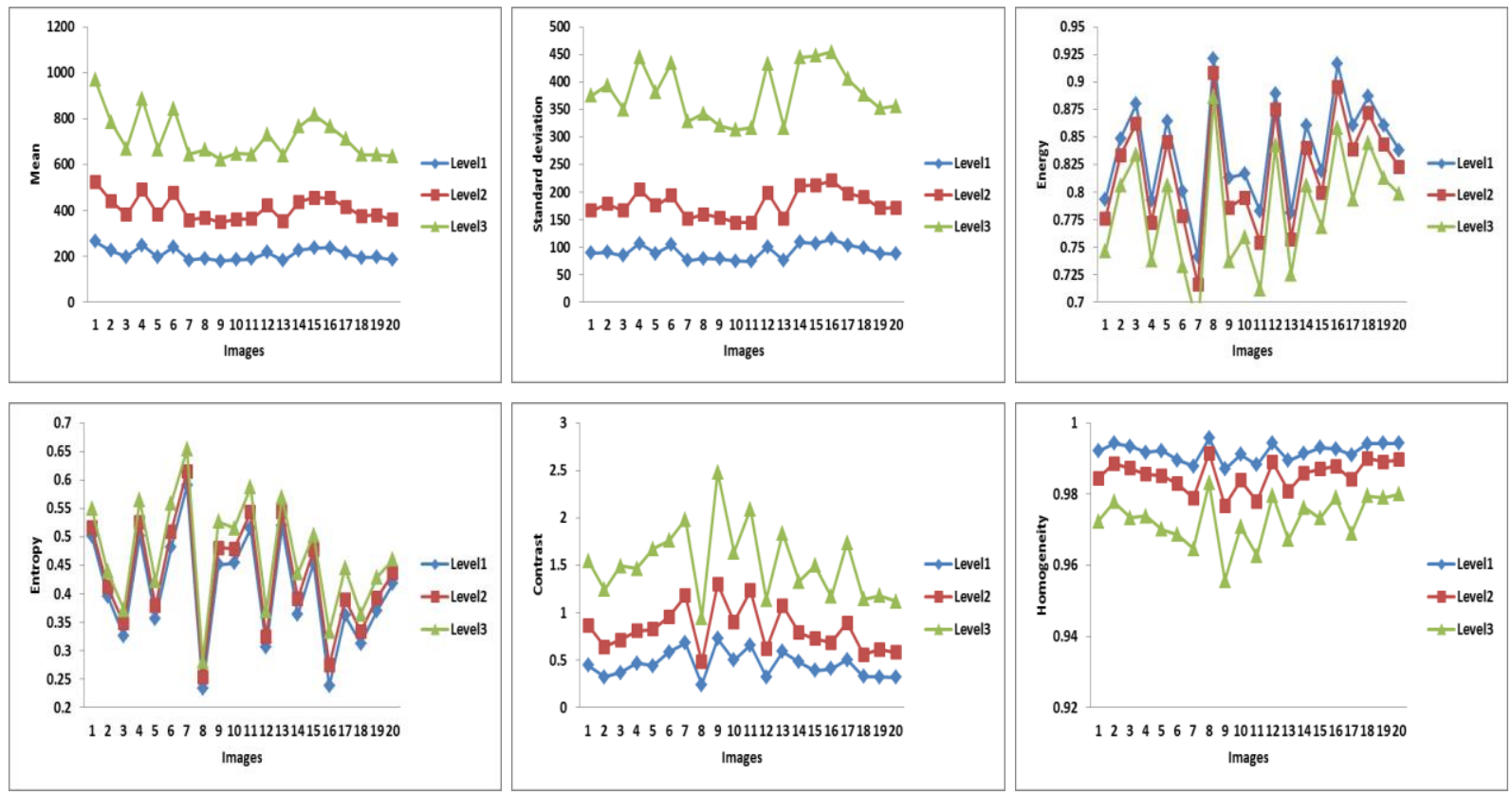

Figure 8: Daubechies wavelet based statistical features obtained for abnormal datasets.

Figures 7 and 8 present the feature statistics obtained by applying the Daubechies wavelet to the segmented region from normal and abnormal slices. It could be noted that all these feature parameters exhibited clear distinction in terms of range of values obtained. 

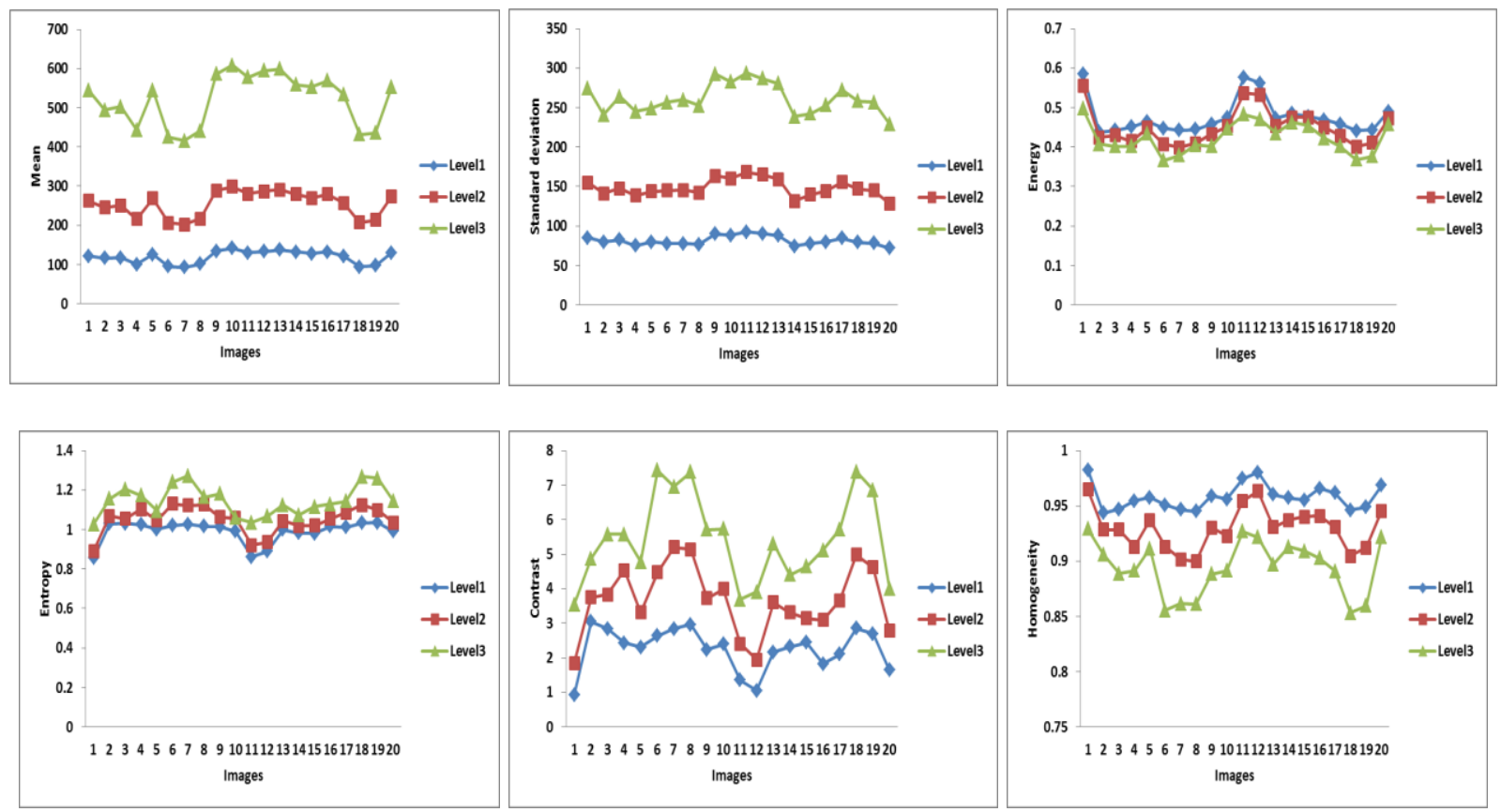

Figure 9: Symlet wavelet based statistical features obtained for normal datasets.
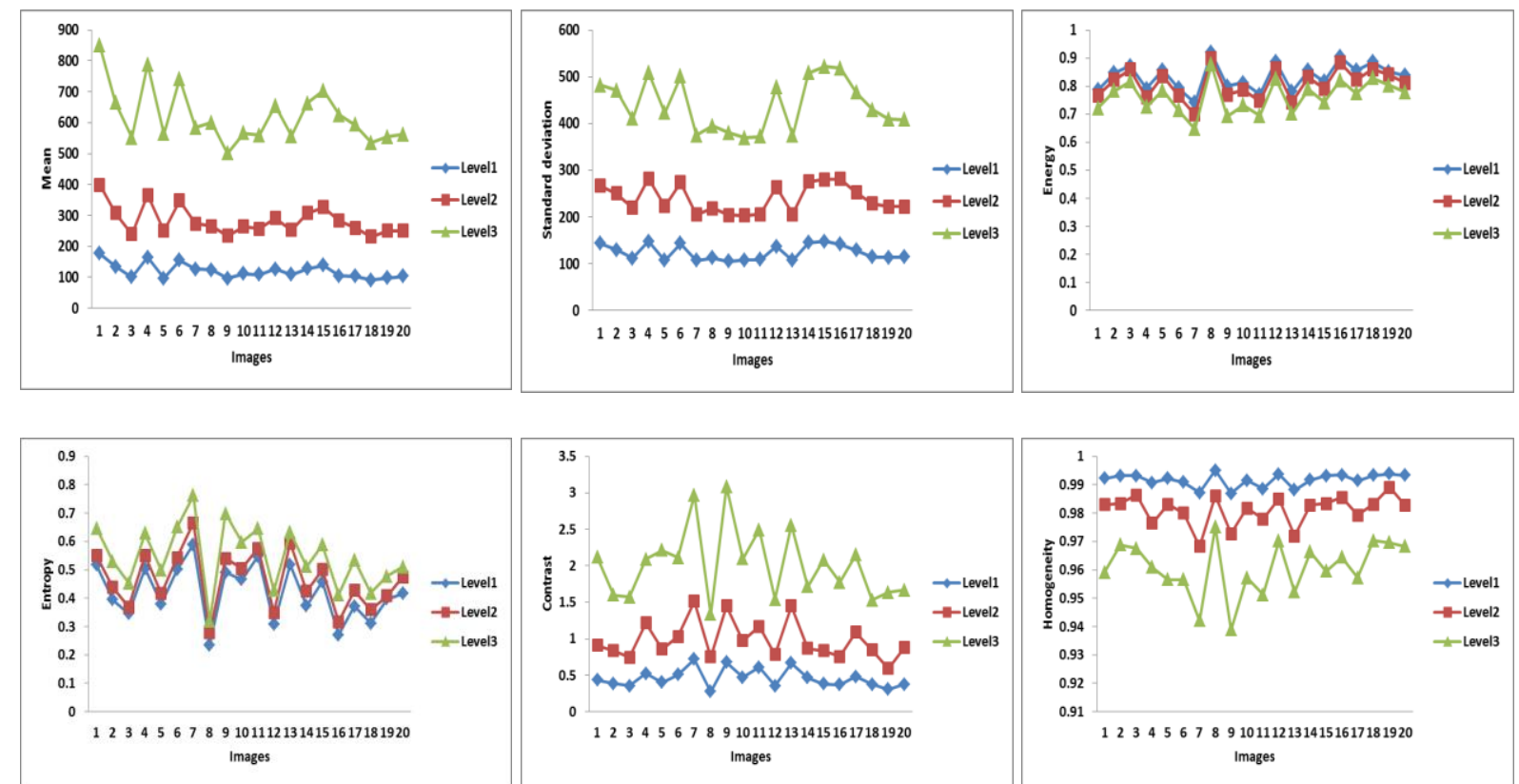

Figure 10: Symlet wavelet based statistical features obtained for abnormal datasets.

Figures 9 and 10 present the feature statistics obtained by applying the Symlet wavelet function to the segmented region from normal and abnormal slices. The energy parameter of the Symlet function exhibited maximum distinction between the normal and abnormal slices. 

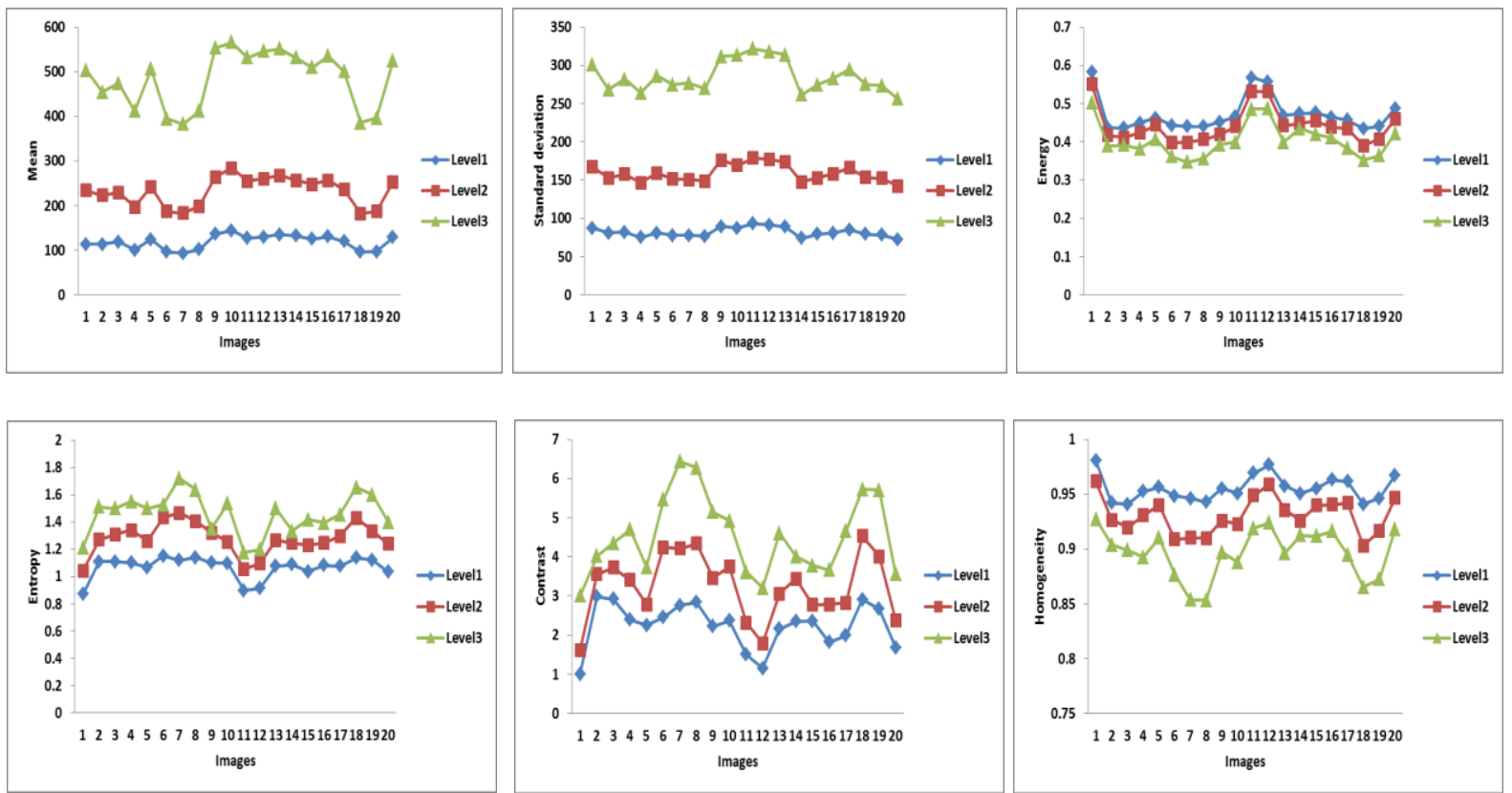

Figure 11: Coiflet wavelet based statistical features obtained for normal datasets.
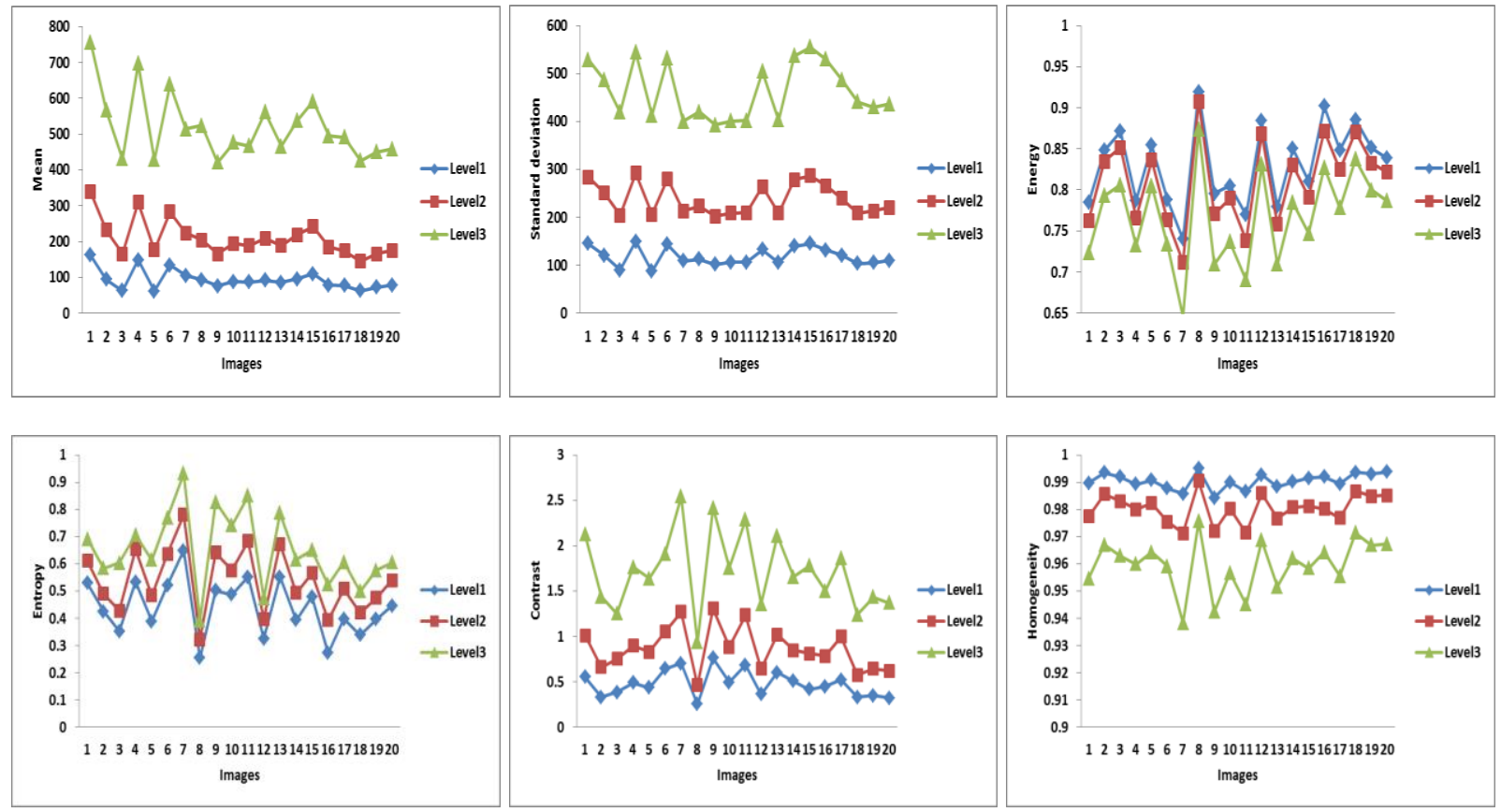

Figure 12: Coiflet wavelet based statistical features obtained for abnormal datasets.

Figures 11 and 12 present the feature statistics obtained by applying the Coiflet wavelet function to the segmented region from normal and abnormal slices. 

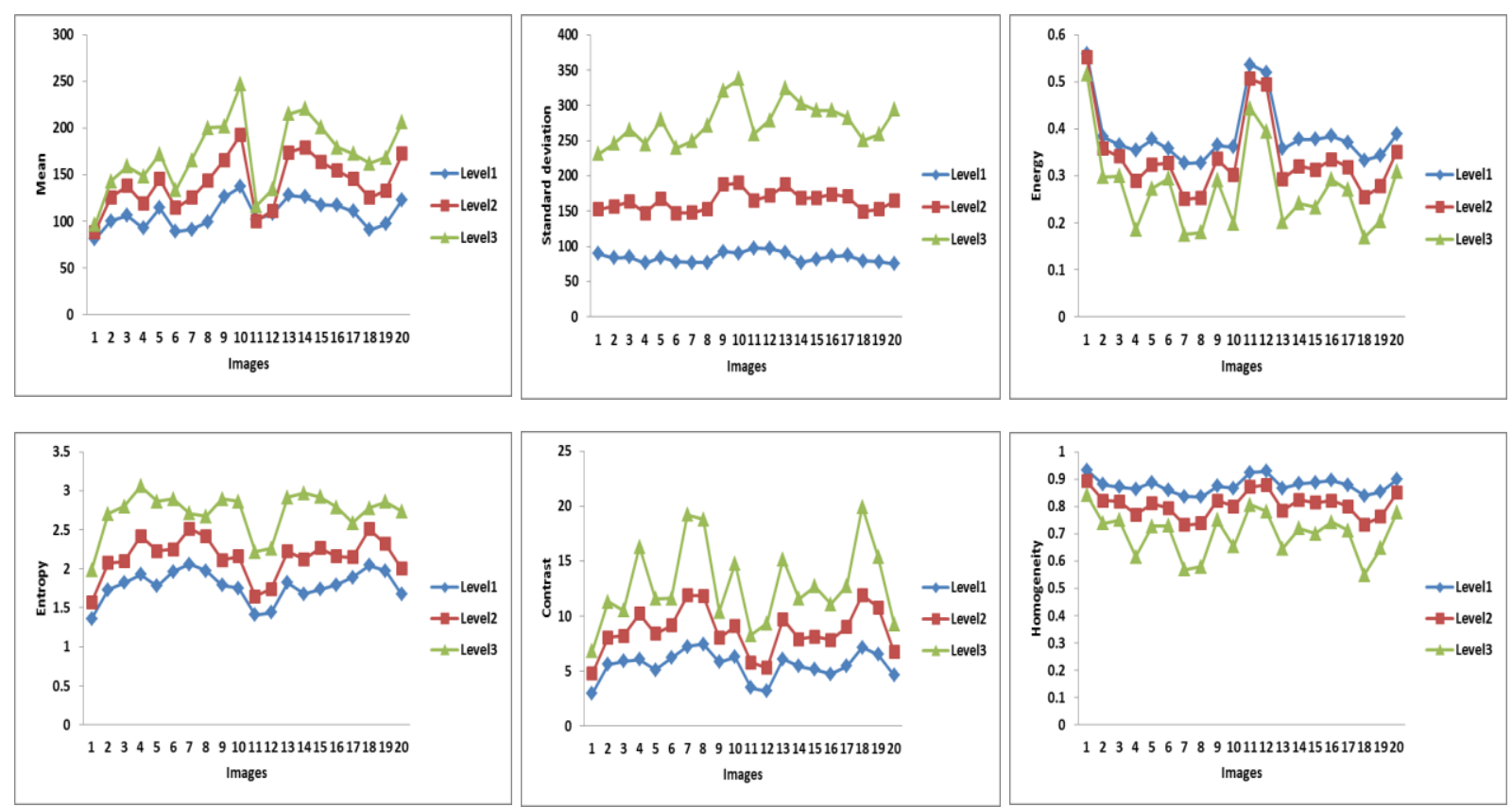

Figure 13: De-Meyer wavelet based statistical features obtained for normal datasets.
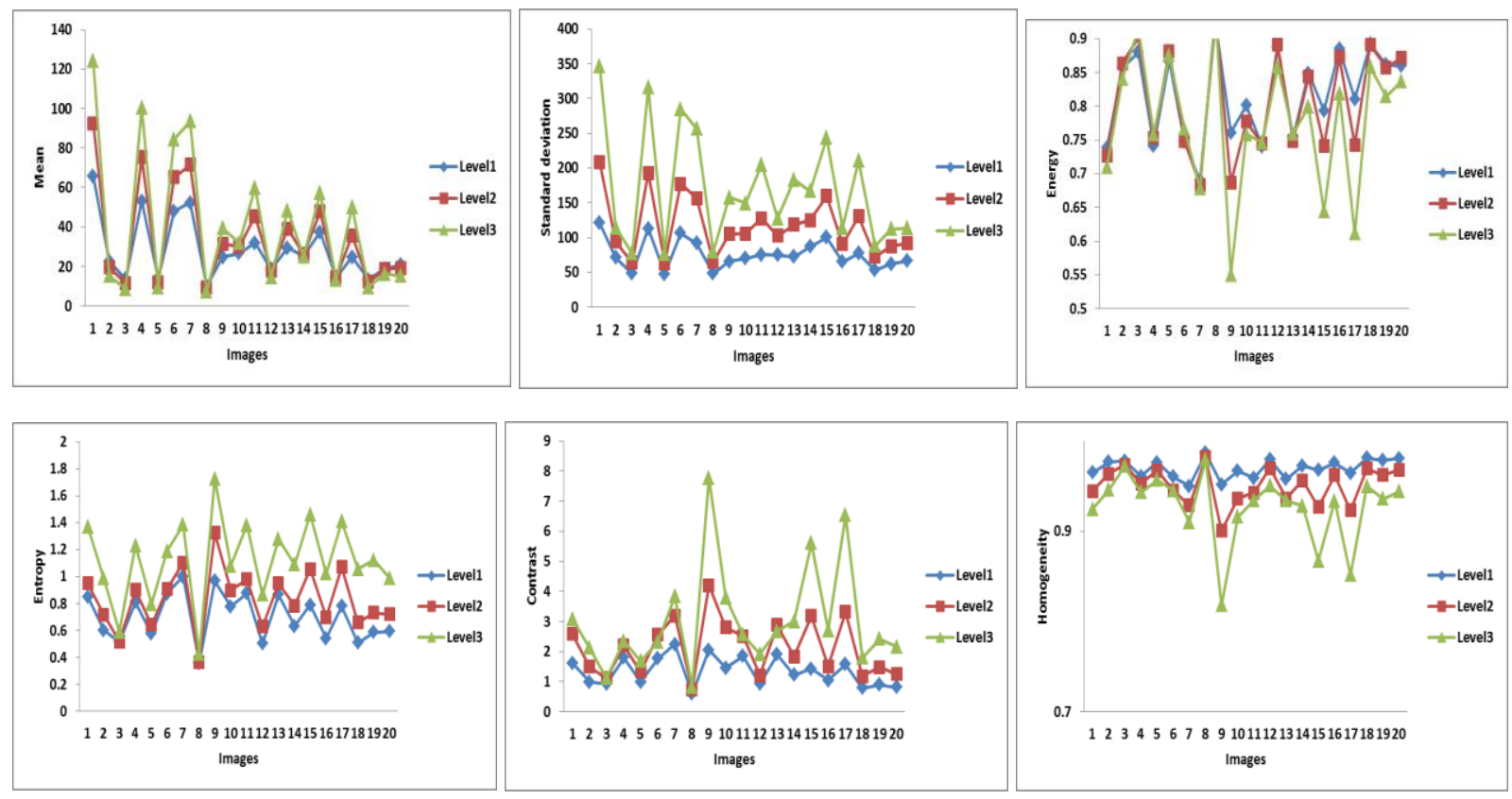

Figure 14: De-Meyer wavelet based statistical features obtained for abnormal datasets.

Figures 13 and 14 present the feature statistics obtained by applying the De-Meyer wavelet function to the segmented region from normal and abnormal slices. The feature parameters namely mean, standard deviation, entropy, contrast and homogeneity parameters were higher for normal brain tissues than the lesion structures for the de-Meyer wavelet function.

The plots presented in Figures 7 to 14 indicate that these features were able to discriminate between normal and abnormal samples. Out of all the features, the energy parameter exhibited maximum discrimination between the two groups. The energy parameter obtained for the lesion structures was relatively high than the normal brain tissues for all the four wavelet functions. The homogeneity parameter was found to be relatively insignificant since it could not provide better discrimination for the normal and abnormal datasets. 


\section{Results and Discussion}

\subsection{Results}

The experiments were carried out on Intel(R) i5 processor. The frequency of the CPU core is $2.3 \mathrm{GHZ}$. The RAM capacity is 4 GB and the execution environment is MATLAB R2012b. Statistical measures namely mean, standard deviation, energy, entropy, contrast and homogeneity were computed for each wavelet function across 3 scales. The cumulative observations based on the feature statistics for different wavelet functions were presented in Tables 1 to 3.

Table 1: Cumulative summary of feature statistics based on mean and standard deviation for all the wavelet-based methods.

\begin{tabular}{|c|c|c|c|c|c|c|c|}
\hline \multirow{2}{*}{ Type of wavelet } & \multicolumn{3}{|c|}{ Mean } & \multicolumn{3}{c|}{ Standard deviation } \\
\cline { 2 - 8 } & Level1 & Level2 & Level3 & Level1 & Level2 & Level3 \\
\hline \multirow{2}{*}{ Daubechies } & Normal & 155.31 & 303.82 & 555.38 & 55.43 & 102.97 & 220.37 \\
\cline { 2 - 8 } & Abnormal & 208.99 & 406.84 & 719.76 & 91.47 & 178.18 & 379.37 \\
\hline \multirow{2}{*}{ Coiflet } & Normal & 118.15 & 232.09 & 483.64 & 81.80 & 159.14 & 286.01 \\
\cline { 2 - 8 } & Abnormal & 93.21 & 209.37 & 519.73 & 118.00 & 238.00 & 463.19 \\
\hline \multirow{2}{*}{ Symlet } & Normal & 119.07 & 254.71 & 520.94 & 81.49 & 148.32 & 261.43 \\
\cline { 2 - 8 } & Abnormal & 119.82 & 282.83 & 620.74 & 123.51 & 239.30 & 439.96 \\
\hline \multirow{2}{*}{ De-Meyer } & Normal & 107.78 & 140.74 & 172.02 & 83.95 & 164.11 & 276.08 \\
\cline { 2 - 8 } & Abnormal & 28.25 & 34.74 & 41.18 & 75.88 & 116.95 & 171.14 \\
\hline
\end{tabular}

Table 2: Cumulative summary of feature statistics based on entropy and energy for all the wavelet-based methods

\begin{tabular}{|c|c|c|c|c|c|c|c|}
\hline \multicolumn{2}{|c|}{ Type of wavelet } & \multicolumn{3}{|c|}{ Entropy } & \multicolumn{3}{c|}{ Energy } \\
\cline { 2 - 8 } & Level1 & Level2 & Level3 & Level1 & Level2 & Level3 \\
\hline \multirow{2}{*}{ Daubechies } & Normal & 0.48 & 0.46 & 0.47 & 0.97 & 0.96 & 0.94 \\
\cline { 2 - 8 } & Abnormal & 0.84 & 0.82 & 0.78 & 0.41 & 0.43 & 0.47 \\
\hline \multirow{2}{*}{ Coiflet } & Normal & 0.47 & 0.44 & 0.40 & 1.07 & 1.28 & 1.46 \\
\cline { 2 - 8 } & Abnormal & 0.83 & 0.81 & 0.77 & 0.44 & 0.54 & 0.65 \\
\hline \multirow{2}{*}{ Symlet } & Normal & 0.48 & 0.45 & 0.42 & 0.99 & 1.05 & 1.15 \\
\cline { 2 - 8 } & Abnormal & 0.83 & 0.81 & 0.76 & 0.42 & 0.46 & 0.55 \\
\hline \multirow{2}{*}{ De-Meyer } & Normal & 0.39 & 0.34 & 0.27 & 1.78 & 2.15 & 2.72 \\
\cline { 2 - 8 } & Abnormal & 0.82 & 0.81 & 0.78 & 0.70 & 0.83 & 1.12 \\
\hline
\end{tabular}

It was observed that the energy parameter of the lesion-based structures was comparatively higher than the normal brain tissues for all the four-wavelet functions. Out of the four different wavelet functions, the Daubechies and De-Meyer wavelets were able to obtain a clear demarcation in the range of feature statistics between the typical brain tissues and abnormal lesion structures. While for the Daubechies wavelet function, the mean, standard deviation, energy and homogeneity parameters was greater for lesion structures than the normal brain tissues, on the other hand, the mean, standard deviation, entropy, contrast and homogeneity parameters were higher for normal brain tissues than the lesion structures for the De-Meyer wavelet function. 
Table 3: Cumulative summary of feature statistics based on contrast and homogeneity for all the waveletbased methods

\begin{tabular}{|c|c|c|c|c|c|c|c|}
\hline \multirow{2}{*}{ Type of wavelet } & \multicolumn{3}{|c|}{ Contrast } & \multicolumn{3}{c|}{ Homogeneity } \\
\cline { 2 - 8 } & Level1 & Level2 & Level3 & Level1 & Level2 & Level3 \\
\hline \multirow{2}{*}{ Daubechies } & Normal & 2.35 & 3.35 & 4.23 & 0.96 & 0.94 & 0.92 \\
\cline { 2 - 8 } & Abnormal & 0.45 & 0.82 & 1.52 & 0.99 & 0.99 & 0.97 \\
\hline \multirow{2}{*}{ Coiflet } & Normal & 2.24 & 3.25 & 4.53 & 0.96 & 0.93 & 0.90 \\
\cline { 2 - 8 } & Abnormal & 0.48 & 0.87 & 1.72 & 0.99 & 0.98 & 0.96 \\
\hline \multirow{2}{*}{ Symlet } & Normal & 2.25 & 3.67 & 5.43 & 0.96 & 0.93 & 0.89 \\
\cline { 2 - 8 } & Abnormal & 0.46 & 0.98 & 2.02 & 0.99 & 0.98 & 0.96 \\
\hline \multirow{2}{*}{ De-Meyer } & Normal & 5.51 & 8.62 & 12.83 & 0.88 & 0.81 & 0.70 \\
\cline { 2 - 8 } & Abnormal & 1.34 & 2.13 & 3.02 & 0.97 & 0.95 & 0.93 \\
\hline
\end{tabular}

These features could be trained using appropriate classifiers to predict the nature of the brain tissues. If it is classified of abnormal type, then the same segmentation procedure could be applied to identify the lesion.

\subsection{Discussion}

The one-way ANOVA statistical test was done in Minitab to check if there is any significant discrimination between the features of normal and abnormal datasets. The test was conducted with $95 \%$ confidence interval and the results obtained were presented Tables 4 to 7 .

Table 4: Statistical significance of features obtained for Daubechies wavelet.

\begin{tabular}{|c|c|c|}
\hline Feature & Level & Statistical Significance \\
\hline \multirow{4}{*}{ Mean } & Level-1 & $P<0.05$ \\
\cline { 2 - 3 } & Level-2 & $P<0.05$ \\
\cline { 2 - 3 } Standard deviation & Level-3 & $P<0.05$ \\
\hline & Level-1 & None \\
\cline { 2 - 3 } & Level-2 & $P<0.05$ \\
\cline { 2 - 3 } & Level-3 & $P<0.05$ \\
\hline \multirow{4}{*}{ Energy } & Level-1 & $P<0.05$ \\
\cline { 2 - 3 } & Level-2 & $P<0.05$ \\
\cline { 2 - 3 } Entropy & Level-3 & $P<0.05$ \\
\hline \multirow{4}{*}{ Contrast } & Level-1 & $P<0.05$ \\
\cline { 2 - 3 } & Level-2 & $P<0.05$ \\
\cline { 2 - 3 } & Level-3 & $P<0.05$ \\
\hline \multirow{3}{*}{ Homogeneity } & Level-1 & None \\
\cline { 2 - 3 } & Level-2 & $P<0.05$ \\
\cline { 2 - 3 } & Level-3 & $P<0.05$ \\
\hline & Level-1 & $P<0.05$ \\
\cline { 2 - 3 } & Level-2 & $P<0.05$ \\
\cline { 2 - 3 } & Level-3 & $P<0.05$ \\
\hline
\end{tabular}

Table 5: Statistical significance of features obtained for Coiflet wavelet. 


\begin{tabular}{|c|c|c|}
\hline Feature & Level & Statistical Significance \\
\hline \multirow{4}{*}{ Mean } & Level-1 & $P<0.05$ \\
\cline { 2 - 3 } & Level-2 & $P<0.05$ \\
\cline { 2 - 3 } Standard deviation & Level-3 & $P<0.05$ \\
\hline & Level-1 & $P<0.05$ \\
\cline { 2 - 3 } & Level-2 & $P<0.05$ \\
\cline { 2 - 3 } Energy & Level-3 & $P<0.05$ \\
\hline \multirow{4}{*}{ Entropy } & Level-1 & $P<0.05$ \\
\cline { 2 - 3 } & Level-2 & $P<0.05$ \\
\cline { 2 - 3 } & Level-3 & $P<0.05$ \\
\hline \multirow{3}{*}{ Contrast } & Level-1 & None \\
\cline { 2 - 3 } & Level-2 & None \\
\cline { 2 - 3 } & Level-3 & $P<0.05$ \\
\hline \multirow{3}{*}{ Homogeneity } & Level-1 & $P<0.05$ \\
\cline { 2 - 3 } & Level-2 & $P<0.05$ \\
\cline { 2 - 3 } & Level-3 & $P<0.05$ \\
\hline & Level-1 & None \\
\cline { 2 - 3 } & Level-2 & None \\
\cline { 2 - 3 } & Level-3 & None \\
\hline
\end{tabular}

Table 6: Statistical significance of features obtained for Symlet wavelet.

\begin{tabular}{|c|c|c|}
\hline Feature & Level & Statistical Significance \\
\hline \multirow{4}{*}{ Mean } & Level-1 & None \\
\cline { 2 - 3 } & Level-2 & None \\
\cline { 2 - 3 } & Level-3 & $P<0.05$ \\
\hline \multirow{3}{*}{$\begin{array}{c}\text { Standard } \\
\text { deviation }\end{array}$} & Level-1 & $P<0.05$ \\
\cline { 2 - 3 } & Level-2 & $P<0.05$ \\
\cline { 2 - 3 } Energy & Level-3 & $P<0.05$ \\
\hline \multirow{4}{*}{ Entropy } & Level-1 & None \\
\cline { 2 - 3 } & Level-2 & $P<0.05$ \\
\cline { 2 - 3 } & Level-3 & $P<0.05$ \\
\hline \multirow{3}{*}{ Contrast } & Level-1 & None \\
\cline { 2 - 3 } & Level-2 & $P<0.05$ \\
\cline { 2 - 3 } & Level-3 & $P<0.05$ \\
\hline & Level-1 & $P<0.05$ \\
\cline { 2 - 3 } & Level-2 & $P<0.05$ \\
\cline { 2 - 3 } & Level-3 & $P<0.05$ \\
\hline \multirow{3}{*}{ Homogeneity } & Level-1 & None \\
\cline { 2 - 3 } & Level-2 & None \\
\cline { 2 - 3 } & Level-3 & $P<0.05$ \\
\hline
\end{tabular}


Table 7: Statistical significance of features obtained for De-Meyer wavelet.

\begin{tabular}{|c|c|c|}
\hline Feature & Level & Statistical Significance \\
\hline \multirow{4}{*}{ Mean } & Level-1 & $P<0.05$ \\
\cline { 2 - 3 } & Level-2 & $P<0.05$ \\
\cline { 2 - 3 } Standard deviation & Level-3 & $P<0.05$ \\
\hline & Level-1 & None \\
\cline { 2 - 3 } & Level-2 & $P<0.05$ \\
\cline { 2 - 3 } Energy & Level-3 & $P<0.05$ \\
\hline \multirow{4}{*}{ Entropy } & Level-1 & $P<0.05$ \\
\cline { 2 - 3 } & Level-2 & $P<0.05$ \\
\cline { 2 - 3 } & Level-3 & $P<0.05$ \\
\hline \multirow{3}{*}{ Contrast } & Level-1 & $P<0.05$ \\
\cline { 2 - 3 } & Level-2 & $P<0.05$ \\
\cline { 2 - 3 } & Level-3 & $P<0.05$ \\
\hline \multirow{3}{*}{ Homogeneity } & Level-1 & None \\
\cline { 2 - 3 } & Level-2 & $P<0.05$ \\
\cline { 2 - 3 } & Level-3 & $P<0.05$ \\
\hline & Level-1 & $P<0.05$ \\
\cline { 2 - 3 } & Level-2 & $P<0.05$ \\
\cline { 2 - 3 } & Level-3 & $P<0.05$ \\
\hline
\end{tabular}

The observations presented in Tables 4 to 7 indicate that the entropy, contrast, homogeneity and standard deviation parameters were not stable in discriminating the samples for most of the wavelet functions. In particular, Table 6 specifies that the symlet wavelet function was not effective in discriminating the normal and abnormal samples due to less statistical significance associated with the features obtained. However, Daubechies and De-Meyer wavelets were able to obtain better discrimination between the samples. Hence, these two wavelets could be used to differentiate the normal and abnormal brain tissues.

\section{Conclusion}

Automatic segmentation of lesion structures from brain MR images serves to identify the level of the influenced tissues for effective diagnosis in the treatment of ischemic stroke. The structure and properties of the lesion structures were generally complex and non-linear in nature. Therefore, it exhibits different characteristics in different imaging modalities. Hence developing a completely automated approach for lesion segmentation is a challenging research issue in medical image analysis. This work examines the properties of the normal brain tissues and abnormal lesion structures by subjecting the brain MR images to three-level wavelet decomposition. Four different wavelet functions namely Daubechies, Symlet, Coiflet and De-Meyer were applied to the different datasets and the resulting observations were clearly analyzed based on their feature statistics obtained. The observations indicate that there was a clear demarcation between the range of values in the statistical features obtained for normal and abnormal images. Specifically, the feature statistics obtained from the Daubechies and De-Meyer wavelets were able to distinguish clearly between the typical brain tissues and abnormal lesion structures based on mean, energy and entropy parameters.

This research was oriented towards the examination of different feature statistics for detection of ischemic stroke disorder. In future, this work can further be enhanced by classifying the stroke into different grades depending on its severity. In addition, it can be extended for characterizing the properties of hemorrhagic stroke lesions. 


\section{References}

[1] J. Byun, M.R. Verardo, B. Sumengen, G.P. Lewis, B.S. Manjunath, S.K. Fisher, "Automated tool for the detection of cell nuclei in digital microscopic images: application to retinal images", Mol. Vis. 12: 949-960, 2006.

[2] C.C. Liao, F. Xiao, J.M. Wong, I.J. Chiang, "A simple genetic algorithm for tracing the deformed midline on a single slice of brain CT using quadratic Bezier curves", Proceedings of Sixth IEEE International Conference on Data Mining Workshops, 2006. DOI: 10.1109/ICDMW.2006.22

[3] T. Thom, N. Haase, W. Rosamond, V.J. Howard, J. Rumsfeld, T. Manolio, et. al., "Heart disease and stroke statistics- 2006 update: a report from the American Heart Association Statistics Committee and Stroke Statistics Subcommittee", Circulation 113: e85-e151,2006. DOI: 10.1161/CIRCULATIONAHA.105.171600

[4] G. H. L. Lemieux, K. Krakow, F. G. Woermann, "Fast, accurate and reproducible automatic segmentation of the brain in weighted volume MRI data", Magn. Reson. Med., 42: 127-135, 1999. DOI: 10.1002/(SICI)1522-2594(199907)42:1<127::AID-MRM17>3.0.CO;2-O

[5] F. Gargouri, Ben Hamida, K. Chtourou, "Automatic localization methodology dedicated to brain tumors based on ICP matching by using axial MRI symmetry", Proc. of the First International Conference on Advanced Technologies for Signal and Image Processing, 2014. DOI: 10.1109/ATSIP.2014.6834608

[6] M. Jafari, S. Kasaei, "Automatic brain tissue detection in MRI images using seeded region growing segmentation and neural network classification", Australian Journal of Basic and Applied Sciences, 5: 1066-79, 2011.

[7] M. Matesin, S. Loncaric, D. Petravic, "A rule-based approach to stroke lesion analysis from CT brain images", Proceedings of the 2nd International Symposium on Image and Signal Processing and Analysis, 2006. DOI: 10.1109/ISPA.2001.938631

[8] S. Shen,W. Sandham, M. Granat, A. Sterr, "MRI fuzzy segmentation of brain tissue using neighborhood attraction with neural-network optimization", IEEE Trans. Inf. Technol. Biomed., 9(3):459-467, 2005. DOI: 10.1109/TITB.2005.847500

[9] D.-Q. Zhang, S.-C. Chen, "A novel kernelized fuzzy C-means algorithm with application in medical image segmentation”,Artif. Intell. Med., 32: 37-50, 2004. DOI: 10.1016/j.artmed.2004.01.012

[10] A. Kassner, R.E. Thornhill, "Texture analysis: a review of neurologic mr imaging applications", Am. J.Neuroradiol, 31:809 -16, 2015. DOI: 10.3174/ajnr.A2061

[11] T.-L. Tan, K.-S. Sim, A.-K. Chong, "Contrast enhancement of CT brain images for detection of ischemic stroke",Proc. of the 2012 International Conference on Biomedical Engineering (ICoBE), 2012. DOI: 10.1109/ICoBE.2012.6179043

[12] M. Chawla, S. Sharma, J. Sivaswamy, L. Kishore, "A method for automatic detection and classification of stroke from brain CT images",Proc. of the 2009 Annual International Conference of the IEEE Engineering in Medicine and Biology Society, 2009. DOI: 10.1109/IEMBS.2009.5335289 
[13] H. Bagher-Ebadian, K. Jafari-Khouzani, P. D. Mitsiaset,M. Lu, H.Soltanian-Zadeh, M.Chopp, J. R. Ewing, "Predicting final extent of ischemic infarction using artificial neural network analysis of multi-parametric MRI in patients with stroke", PloS One, 6:e22626, 2011. DOI: 10.1371/journal.pone.0022626

[14] P. Dastidar, T. Heinonen, J.P. Ahonen, M. Jehkonen, G. Molnár, "Volumetric measurements of right cerebral hemisphere infarction: use of a semiautomatic MRI segmentation technique", Comput. Biol. Med., 30: 41-54, 2000. DOI: 10.1016/S0010-4825(99)00022-0

[15] N.Nabizadeh, M.Kubat, "Brain tumors detection and segmentation in MR images: Gabor wavelet vs. statistical features", Computers and Electrical Engineering, 45:286-301, 2015. DOI: 10.1016/j.compeleceng.2015.02.007

[16] S. Yang, S. Mitra, E. Corona, B. Nutter, D. J. Lee, "Multilevel wavelet feature statistics for efficient retrieval, transmission, and display of medical images by hybrid encoding", EURASIP Journal on Applied Signal Processing, 5:449-460, 2003. DOI: 10.1155/S1110865703211203

[17] S. Y. Yeo, "Implicit active contours for N-dimensional biomedical image segmentation", Proceedings of the IEEE International Conference on Systems Man, and Cybernetics, 2012. DOI: 10.1109/ICSMC.2012.6378182

[18] Q. Ge, C. Li, W. Shao, H. Li, "A hybrid active contour model with structured feature for image segmentation”, Signal Processing. 108:147-58, 2015.DOI: 10.1016/j.sigpro.2014.09.007

[19] Q. Wu, Y. Gan, B. Lin, Q. Zhang, H. Chang, "An active contour model based on fused texture features for image segmentation", Neurocomputing. 151(3):1133-41, 2015.DOI: 10.1016/j.neucom.2014.04.085

[20] A. Rajendran, R. Dhanasekaran, "Fuzzy clustering and deformable model for tumor segmentation on MRI brain image: A combined approach", Procedia Engineering, 30: 327-33, 2011. DOI: 10.1016/j.proeng.2012.01.868.

[21] R. Karthik., Menaka, R., Chellamuthu, C. (2015) 'A comprehensive framework for classification of brain tumour images using SVM and curvelet transform', Int. J. Biomedical Engineering and Technology, Vol. 17, No. 2, pp.168-177. DOI: 10.1504/IJBET.2015.068054

[22] https://www.virtualskeleton.ch/ISLES/Start2015

[23] http://www.isles-challenge.org/

[24] I. Maiti, M. Chakraborty, "A new method for brain tumor segmentation based on watershed and edge detection algorithms in HSV colour model", National Conference on Computing and Communication Systems, 2012. DOI: 10.1109/NCCCS.2012.6413020

[25] I. Daubechies, "Ten lectures on wavelets", Proc. CMBS-NSF Regional Conference Series in Applied Mathematics,1992.DOI: 10.1137/1.9781611970104

[26] A. Pizurica, W. Philips, "A versatile wavelet domain noise filtration technique for medical imaging”, IEEE Trans. Med. Imag, 22(3):323-331, 2003. DOI : 10.1109/TMI.2003.809588 